\title{
Semen analysis - An evaluation of 170 cases in rural India
}

\author{
Rahul Jhadav $^{1}$, Sheetal Mahajan ${ }^{2, *}$, K.S. Bhople ${ }^{3}$ \\ ${ }^{1,2}$ Assistant Professor, ${ }^{3}$ Professor and Head, Dept. of Pathology, Indian Institute of Medical Sciences and Research, Jalna, \\ Maharashtra, India
}

*Corresponding Author: Sheetal Mahajan

Email: rahulmankura@gmail.com

\begin{abstract}
Introduction: Semen Analysis, despite its limitations, remains the single most important test for evaluating male factor of infertility. The methods for evaluation of male infertility typically have been limited to a semen analysis that evaluates sperm count, motility, and morphology. Recently WHO have set lower reference limits for the examination and processing of human semen in 2010.

\section{Aims and Objectives}

1. To evaluate male factor of infertility with help of WHO laboratory manual for semen analysis.

2. To know the role of addiction in deranged semen analysis among males in rural population.

Materials and Methods: The study was conducted in Indian Institute of Medical Sciences and Research, Warudi Tq Badnapur, Dist Jalna from $1^{\text {st }}$ of February 2017 to $1^{\text {st }}$ February 2018. A cross sectional study of 170 cases of semen analysis was carried out in details during period of 1 year. A detailed history of patient was taken, history of addiction was noted and reporting was done as per WHO guidelines.

Results: Total 170 cases were studied.17.6\% cases had low semen volume. $12.9 \%$ cases had prolonged liquefaction time. $35.3 \%$ patients had oligozoospermia or azoospermia. Motility was affected in $45.9 \%$ cases and viability in $11.7 \%$ cases. Morphology was affected in $10.3 \%$ cases. $73.4 \%$ cases show some kind of addiction.

Conclusion: Motility is the commonest factor found deranged in semen analysis in our study. Some forms of morphological defects are common in semen samples and should be noted. Addiction plays important role in deciding fertility of the patient and should be given due importance.
\end{abstract}

Keywords: Male infertility, Semen analysis.

\section{Introduction}

A semen analysis is still the test of choice for assessment of male partner in an infertile couple. Studies that link quality of ejaculate and fertility started since the 1930. ${ }^{1}$ World Health Organization (WHO) manuals have served as a primary reference for seminal fluid analysis procedures since long. WHO has published five editions of the manual for semen analysis at different times and the most recent $5^{\text {th }}$ edition was published in 2010. The standards \& reference value for different parameters have been defined again with each subsequent new edition. According to us, initial 4 editions were based on consensus by experts but fifth edition is evidence based and hence superior to initial 4 editions. Semen is formed in testis and consists of two components. The $1^{\text {st }}$ is spermatozoa made by the seminiferous tubules of the testis, and the $2^{\text {nd }}$ is seminal fluid produced by the accessory glands. This fluid nourishes sperm and plays a role in interacting with the female reproductive tract to influence fertility. ${ }^{2}$ These components are reflected in the semen analysis by the gross and microscopic examination (Volume and sperm count). The total sperm count reflects the number of spermatozoa in the semen sample. The sperm concentration is the number of sperms per $\mathrm{ml}$ of semen. The volume of the semen reflects the amount of seminal fluid produced by accessory glands. ${ }^{3}$ According to WHO, semen volume, sperm concentration, total sperm number, morphology, motility and vitality are most important factors for male fertility. The aim of present study is to discuss the role and limitations of semen analyses for evaluating of infertile couple, as well as to find the correlation of addiction with infertility and deranged semen parameters in infertile couple with respect to rural population.

Semen parameters vary from time to time in the same individual like any other fluid parameters. ${ }^{4}$ Semen sample is collected by masturbation with an abstinence period of two to seven days, usually near the laboratory premises to reduce the time interval between collection and analysis of sample as the parameters quickly change over time. The gross findings of the semen sample, such as the volume of semen, $\mathrm{pH}$ of semen, color of semen, liquefaction time and viscosity is measured carefully. The sample is then further evaluated under a light microscope to determine the motility of sperms, vitality of sperms, concentration of sperms, and morphology of sperms..$^{5}$ According to current WHO manual 2010, semen volume more than $1.5 \mathrm{ml}$, sperm concentration more than 15 millions, progressive motility more than $32 \%$, more than $58 \%$ live forms with more than $4 \%$ normal forms defines what we call reference range for semen analysis. ${ }^{3}$ Many studies have proved that the total motile sperm count (volume $\mathrm{x}$ concentration $\mathrm{x}$ motility) is the most predictive factor in assessing fertility of male as 
compared to volume of semen, concentration of sperms, and motility of sperms counted individually. ${ }^{6}$

There are many studies on semen analysis. A review study by Wang and Swerdloff (2014) discussed problems of using semen analysis as a test for male infertility and emphasized the importance of seeing the problem of infertility as issue of couple rather than of individual. This study also discussed the importance of semen analysis in azoospermia. ${ }^{7}$ Dr N Pandiyan in his article 'Semen analysis-A number game' concluded that there is need to redefine normal semen parameters and population based studies are essential to establish normal values. ${ }^{4}$ Patel et al concluded that impaired parameters in semen analysis alone cannot be used to predict fertility of males. These men still have a good chance of being fertile with few exceptions. ${ }^{8}$ Kruger in 2016 concluded that semen deranged semen parameters in case of varicocele will improve after varicocelectomy. ${ }^{9}$

\section{Objective}

To analyze semen parameters according to WHO criteria in patients attending infertility OPD. To find correlation between semen parameters and addiction among males in rural population.

\section{Materials and Methods}

This study was done in clinical pathology section of pathology department in rural based medical college, Indian Institute of Medical Sciences and Research, Warudi Dist. Jalna (Maharashtra). This was a prospective study conducted during period of one year from $1^{\text {st }}$ of February 2017 to $1^{\text {st }}$ February 2018. Total 170 couples reaching infertility OPD were considered for this study. The female partners were evaluated for infertility. Only those male patients were considered for the study whose partners were not having any abnormality in fertility evaluation. These men were called for semen analysis after 3 days of abstinence. The semen samples were collected in laboratory in clean sterile containers by masturbation. These samples were then processed for gross and microscopic examination. Detailed history like addiction, occupation, history of previous issue was noted. All patients with past history of smoking or present smokers were considered as smokers. Presence of varicocele was noted. A systematic report was made for each patient according to WHO manual for semen analysis. Gross examination was noted as volume, color, PH, liquefaction time and viscosity. For microscopic examination, wet mount study, Neubauer's chamber for counting and eosin-nigrosin smears for vitality was done. Results obtained were tabulated and analyzed.

\section{Results}

As discussed earlier, volume of semen is formed by two major constituents, spermatozoa and fluid secreted by seminal vesicles. The volume of the ejaculate is contributed mainly by the seminal vesicles and prostate gland, with a small amount from the bulbourethral glands and epididymides. Precise measurement of volume is essential in any evaluation of semen, because it allows the total number of spermatozoa and nonsperm cells in the ejaculate to be calculated. ${ }^{3}$ WHO considers $1.5 \mathrm{ml}$ as the lower limit for semen volume and lower values are seen in obstruction of the ejaculatory duct, congenital bilateral absence of the vas deferens (CBAVD), retrograde ejaculation or collection problems. ${ }^{3}$ In our study, $82.4 \%$ cases showed normal semen volume while $17.6 \%$ cases showed reduced semen volume (Table 1). The problems associated with collection can be reduced by counseling patient before the collection of sample. In case of retrograde ejaculation, a post ejaculate urine examination is necessary for detection of sperms in urine.

Appearance or color of semen has some significance in semen examination. Normally liquefied semen show homogeneous, grey-opaque appearance and appear less opaque if the sperm concentration is low. The red color is seen in haemospermia and yellow color in a man with jaundice or taking certain vitamins/drugs or more abstinence period. ${ }^{3}$ In our study, $61.8 \%$ samples show normal white opaque appearance which correlated with number of samples with normal sperm count (64.7\%). 26.5\% samples show less opaque watery appearance and $11.7 \%$ samples show slightly yellowish tinge to semen sample. None of the samples showed reddish appearance (Table 2).

When collected, semen appear as mass of thick coagulum. After few minutes, prostatic proteases liquefy semen and it appears watery. This usually happens within $15 \mathrm{~min}$ or sometimes takes upto $60 \mathrm{~min}$. The initial part of semen is rich in sperms and prostatic fluid while later part is rich in seminal vesicular fluid. Hence, the initial part of semen is very important to correctly calculate liquefaction time. Delayed liquefaction ( $>60 \mathrm{~min}$ ) is noted and indicate pathology. These samples usually show high viscosity. In our study, 148 patients $(87.1 \%)$ had normal liquefaction time and 22 patients (12.9\%) had deranged liquefaction time. High viscosity can be made out by the elastic property of sample, which adheres strongly to itself when tried to pipette it. High viscosity is likely to interfere with sperm motility and sperm concentration. ${ }^{3}$

Sperms are counted by using semen diluting fluid on Neubauer's chamber. Sperm concentration $<15$ million $/ \mathrm{ml}$ is considered as lower limit by WHO. A sperm concentration below $15 \mathrm{million} / \mathrm{ml}$ is regarded as oligozoospermia. In our study, $20.6 \%$ patients had oligozoospermia while $14.7 \%$ patients had azoospermia. $64.7 \%$ patients had normal sperm concentration. It is important indicator of male fertility. Aggregation is a non specific entity and refers to sticking non motile sperms to each other or to other cells or debris. Agglutination specifically refers to 
motile sperms sticking to each other from head-to-head, tail-to-tail or in a mixed way. Both should be noted.

The progressive motility is important indicator of male fertility and is related to pregnancy rates. WHO has given guidelines for motility evaluation in a semen sample. A Progressive motility is defined as spermatozoa moving actively, either linearly or in a large circle, regardless of speed. All other forms are considered as non progressive motility while non moving sperms are classified as immotile. In our study, $45.9 \%$ patients had $<32 \%$ progressive motility and $54.1 \%$ patients had normal progressive motility.

Sperm vitality is the assessment of membrane integrity. It is important to know whether immotile sperms are alive or dead. Vitality results were assessed in conjunction with motility results from the same semen sample. We used dye exclusion method which is based on the principle that damaged plasma membranes, such as those found in dead sperms cells, allow entry of membrane-impermeable stains. In our study, $88.3 \%$ patients had $>58 \%$ viable sperms and rest had reduced viable sperms.

Morphology of sperms is very important clue for male fertility. Many morphological abnormalities are seen in sperms like abnormality of head, middle piece and tail. Some form of abnormality is seen in almost all semen samples. But it is the count of abnormal cells that matters. ${ }^{10}$ An arbitrary limit of $4 \%$ is set by WHO for abnormal sperm cells. $<4 \%$ normal sperm cells are significant and labelled as teratozoospermia.

Presence of round cells in semen may be due to leucocytes or immature germ cells. $17.6 \%$ cases showed increased number of round cells in semen sample.

Of 170 patients, 45 had no addiction history in past or present. They constituted $26.6 \%$ of all cases. Rest 73.4\% patients gave some kind of addiction history of smoking, tobacco or alcohol. $23.5 \%$ patients had addiction for all 3 kind of stuff. 40 patients were addicted to smoking alone. (Table 9)

Table 1: Volume of semen per case per ejaculate

\begin{tabular}{|l|c|c|}
\hline \multicolumn{1}{|c|}{ Volume } & Cases & \% \\
\hline$<1.5 \mathrm{ml}$ & 030 & 17.6 \\
\hline$>1.5 \mathrm{ml}$ & 140 & 82.4 \\
\hline Total & 170 & 100 \\
\hline
\end{tabular}

Table 2: Color of semen

\begin{tabular}{|l|c|c|}
\hline \multicolumn{1}{|c|}{ Color } & Cases & \% \\
\hline White opaque & 105 & 61.8 \\
\hline Less opaque & 045 & 26.5 \\
\hline Yellowish & 020 & 11.7 \\
\hline Reddish & 0 & 0 \\
\hline Total & 170 & 100 \\
\hline
\end{tabular}

Table 3: Liquefaction time of semen

\begin{tabular}{|l|c|c|}
\hline \multicolumn{1}{|c|}{ Time } & Cases & \% \\
\hline$<30 \mathrm{~min}$ & 148 & 87.1 \\
\hline$>60 \mathrm{~min}$ & 022 & 12.9 \\
\hline Total & 170 & 100 \\
\hline
\end{tabular}

Table 4: Sperm concentration in semen

\begin{tabular}{|l|c|c|}
\hline \multicolumn{1}{|c|}{ Count } & Cases & \% \\
\hline$>15$ million & 110 & 64.7 \\
\hline$>0$ and $<15$ million & 35 & 20.6 \\
\hline No sperms & 25 & 14.7 \\
\hline Total & 170 & 100 \\
\hline
\end{tabular}

Table 5: Progressive motility of sperms

\begin{tabular}{|l|c|c|}
\hline \multicolumn{1}{|c|}{ Motility } & Cases & \% \\
\hline$>32 \%$ progressive & 92 & 54.1 \\
\hline$<32 \%$ progressive & 78 & 45.9 \\
\hline Total & 170 & 100 \\
\hline
\end{tabular}

Table 6: Viability of sperms

\begin{tabular}{|l|c|c|}
\hline \multicolumn{1}{|c|}{ Viability } & Cases & \% \\
\hline$>58 \%$ & 128 & 88.3 \\
\hline$<58 \%$ & 17 & 11.7 \\
\hline Total & 145 & 100 \\
\hline
\end{tabular}

Table 7: Morphology of sperms

\begin{tabular}{|l|c|c|}
\hline Morphology & Cases & \% \\
\hline$>4 \%$ Normal forms & 130 & 89.7 \\
\hline$<4 \%$ Normal forms & 15 & 10.3 \\
\hline Total & 145 & 100 \\
\hline
\end{tabular}

Table 8: Round cell concentration

\begin{tabular}{|l|c|c|}
\hline \multicolumn{1}{|c|}{ Round cells } & Cases & \% \\
\hline Increased & 30 & 17.6 \\
\hline Normal & 140 & 82.4 \\
\hline
\end{tabular}

Table 9: Addiction in patients

\begin{tabular}{|c|c|c|c|}
\hline Addiction & Cases & $\%$ & $\%$ \\
\hline Smoking & 40 & 23.5 & \multirow{4}{*}{73.4} \\
\hline Tobbacco & 30 & 17.6 & \\
\hline Alcohole & 15 & 8.8 & \\
\hline All of above & 40 & 23.5 & \\
\hline No addiction & 45 & 26.6 & 26.6 \\
\hline Total & 170 & 100 & 100 \\
\hline
\end{tabular}

\section{Discussion}

Infertility is a mental and financial trauma to a couple who wish children. Semen analysis is an important tool for assessment of male factor of infertility. Causes of male infertility are numerous. Varicocele, trauma, obstruction, undescended testis are some of them while some causes remain unknown. There are many studies that link prenatal exposure of smoking to sperm quality in offspring resulting in infertility. Jensen TK et al in 2004 studied association of in utero exposure to maternal smoking with reduced 
semen quality and testis size in adulthood in 1,770 young men from Europe. ${ }^{11}$

The presence of round cells in semen may be suggestive of testicular damage (immature germ cells) or inflammation of accessory glands (leukocytes). Excess numbers of leukocytes in the semen (leukocytospermia) may be associated with infection and poor sperm quality. Leukocyte-dependent damage to sperms depends on the total leukocyte number in the semen and the number of leukocytes compared to the number of sperms. The concentration of round cells is calculated relative to spermatozoa by assessing stained semen smears made from undiluted semen. If $\mathrm{N}$ is the number of round cells counted in the same number of fields as four hundred sperms, and $\mathrm{S}$ is concentration of sperms $\left(10^{6}\right.$ per $\left.\mathrm{ml}\right)$, then the concentration $(\mathrm{C})$ of round cells $\left(10^{6}\right.$ per $\left.\mathrm{ml}\right)$ can be easily calculated from the formula $\mathrm{C}=\mathrm{S} \times(\mathrm{N} / 400)$. The total number of round cells in given ejaculate may reflect the severity of the inflammatory or spermatogenic condition. This is easily obtained by multiplying the concentration of round cells by volume of the whole ejaculate. ${ }^{3}$ In our study, round cells were seen increased in $17.6 \%$ cases. In a study by Trummer et al in 2002 in their article 'The impact of cigarette smoking on semen parameters and hormones' concluded that smoking does not affect conventional semen parameters but significantly increases round cells and leucocytes in semen ${ }^{12}$. These findings partially correlate with our study.

Lindholm et $\mathrm{al}^{13}$ in 1978 and Willis et a ${ }^{14}$ in 1983 demonstrated toxic effects of high doses of ethanol on the testes of man and experimental animals. In our study $32.3 \%$ patient gave history of long term alcoholism. The amount of daily alcohol consumption could not be elaborated in our study but the number of patient associated with alcoholism and attending infertility clinic was significant. Hence findings of our study matches with the above mentioned studies.

In few studies, smoking was associated with abnormal morphology of sperms. Goyal et $\mathrm{al}^{15}$ in their article 'qualitative defects with normal sperm counts in a patient attending infertility clinic' studied sperm morphological defects in patients with normal sperm count. They concluded that qualitative defects are seen even in cases with normal sperm counts. The most common abnormality found was head abnormalities followed by middle piece abnormalities and least common were tail abnormalities. In study by Sharma et al, ${ }^{16}$ tail abnormalities are increased in smokers. In our study, morphological abnormalities were seen in all patients. This finding supports the study by Goyal et al. But significant morphological abnormality (>96\% abnormal sperms) is seen only in $10.3 \%$ patients. So it is very difficult to establish a relationship between smoking and significant morphological sperm abnormalities from above findings and further studies are needed to confirm this association. Presence of increased number of patients with abnormal sperm morphology provides indirect evidence of its association with smoking.

To summarize, we can say that semen analysis is a promising tool for clinicians to judge fertility of patients. Semen analysis has evolved over the period and WHO guidelines provide useful data to assess the semen of patient. As new factors affecting the fertility of male are being uncovered, we must look ahead and search for new tools for semen analysis. This study was an attempt to analyze fertility affecting factors in rural population.

\section{Conclusion}

Semen analysis is a very important tool for evaluation of male infertility and in expert hand, it will provide vital information to clinician. Abnormal morphology of sperms should be noted precisely. History of addiction provides important clue to pathologist during semen analysis and should not be neglected.

\section{References}

1. Macomber D, Sanders MB. The spermatozoa count. $N$ Engl J Med. 1929;200:984-7.

2. Schjenken JE, Robertson SA. Seminal fluid signalling in the female reproductive tract: implications for reproductive success and offspring health. Adv Exp Med Biol. 2015;868:127-58.

3. World Health Organization Department of Reproductive Health and Research. WHO laboratory manual for the examination and processing of human semen, fifth edition. WHO 2010.

4. Dr. N. Pandiyan. Semen Analysis - A Numbers Game. Chettinad Health City Medical Journal. 2015.1-3

5. 5 Esteves SC, Zini A, Aziz N, Alvarez JG, Sabanegh Jr ES, Agarwal A. Critical appraisal of World Health Organization's new reference values for human semen characteristics and effect on diagnosis and treatment of subfertile men. Urology. 2012;79:16-22.

6. Bonde JP, Ernst E, Jensen TK, Hjollund NH, Kolstad H, Henriksen TB, et al. Relation between semen quality and fertility: a population-based study of 430 first-pregnancy planners. Lancet. 1998;352:1172-7.

7. Wang C, Swerdloff RS. Limitations of semen analysis as a test of male fertility and anticipated needs from newer tests. Fertil Steril. 2014;102:1502-7.

8. Patel. Prediction of male infertility by the World Health Organization laboratory manual for assessment of semen analysis: A systematic review. Arab Journal of Urology. 2018:16;96-102.

9. Kruger T. Critical appraisal of conventional semen analysis in the context of varicocele. Asian Journal of Andrology. 2016;18:202-204.

10. Goyal R, Kotru M, Gogia A, Sharma S. Qualitative defects with normal sperm counts in a patient attending infertility clinic. Indian J Pathol Microbiol. 2018;61:2335.

11. Jensen TK, Jorgensen N, Punab M, Haugen TB, Suominen J, Zilaitiene B, Horte A, Andersen AG, Carlsen E, Magnus $\varnothing$ et al. Association of in utero exposure to maternal smoking with reduced semen quality and testis size in adulthood: a cross-sectional study of 1,770 young men from the general population in five European countries. Am J Epidemiol. 2004;159:49-58. 
12. Trummer H, Habermann H, Haas J, Pummer K. The impact of cigarette smoking on human semen parameters and hormones. Hum Reprod. 2002;17:1554-1559.

13. Lindholm J, Fabricius-Bjerre N, Bahnsen M, Boisen P, Bangstrup L, Pedersen M L \& Hagen C (1978): Pituitarytesticular function in patients with chronic alcoholism. Eur J Clin Invest. 8:269.

14. Willis B R, Anderson R A, Oswald C \& Zaneveld L J D (1983): Ethanol-induced male reproductive tract phatology as a function of ethanol dose and duration of exposure. J Pharmacol Exp Ther. 225:470.

15. Goyal R, Kotru M, Gogia A, Sharma S. Qualitative defects with normal sperm counts in a patient attending infertility clinic. Indian J pathol and microbiol. 2018;61:233-5.

16. Singh S, Sharma S, Jain M, Chauhan R. Importance of papanicolaou staining for sperm morphologic analysis: Comparison with an automated sperm quality analyzer. Am J Clin Pathol. 2011;136:247-51. 